\title{
Ventajas económicas y ambientales para el aprovechamiento del potencial industrial de cogeneración en México
}

\author{
G. León de los Santos \\ División de Estudios de Posgrado \\ Facultad de Ingeniería, UNAM \\ E-mail:tesgleon@aol.com
}

(recibido: abril de 2002; aceptado: septiembre de 2002)

\section{Resumen}

México tiene un importante potencial de cogeneración industrial que no ha sido evaluado ni proyectado dentro de su crecimiento histórico, por ello, el objetivo de este trabajo consiste en estudiar y conocer los ahorros económicos y ambientales que se podrían lograr si se aprovechara dicho potencial.

Como resultado, se muestra que la cogeneración en México ofrece un potencial teórico medio de 28,000 MWe al año 2007. Se estima también un ahorro económico en la construcción de infraestructura de generación eléctrica para el escenario alto de su aprovechamiento de casi 7000 mdd, y un escenario de reducción de emisiones para el sector industrial del país de un $0.35 \%$ a un $21.47 \%$ para el año 2007. Estos pronósticos se pueden lograr, siempre y cuando el gobierno cambie la visión de manejar este tema como una medida de ahorro de energía, y la sustituya por la política de "complemento al desarrollo del sector eléctrico nacional".

Descriptores: cogeneración, potencial, costos, ahorros, mitigación, viabilidad.

\section{Abstract}

Mexico has an industrial cogeneration potential very important, not evaluated or projected within its historical growth. The objective of the present work consist of studying the industrial cogeneration potential that exists in Mexico, as well as the economic and environmental savings that it would be achieved if one took advantage of this potential.

As a result of the present work we can conclude that the cogeneration in Mexico offers a theorstical potential middle of 28,000 MWe to the 2007. It is estimated an economic saving potential in the construction of generation infrastructure electrical, for the high stage of its utilization of almost 7000 million of dollars and a stage of emissions reduction of $2007-21.47 \%$, instead of 2007 $0.35 \%$, for the industrial sector of the country. Provided that change the vision of the government of seeing to the cogeneration as a measure of energy saving, and substitute it by that of political of complement to the development of the national electrical sector.

Keywords: cogeneration (CHP), potential, savings, mitigation, viability. 


\section{Introducción}

El desarrollo de la infraestructura eléctrica que requerirá en la próxima década el país, representa un reto para el gobierno, principalmente para la Comisión Federal de Electricidad (CFE); dado el crecimiento del monto de inversión requerida para la construcción de esta infrastructura, la cual demanda tasas (PSEN, 1997) de 5.8 a $5.9 \%$ anuales durante los 10 años siguientes.

La cogeneración en la industria le ofrece a la CFE que por cada Megawatt eléctrico (MWe) cogenerado, se deje de demandar esa misma cantidad en la red, así, la empresa eléctrica queda en posibilidades de destinar esa capacidad a otro consumidor.

En cuanto a la evaluación financiera, la viabilidad económica del potencial industrial de cogeneración, presenta una distribución económica dada por la mejora en la eficiencia global de generación, respecto a la forma convencional lograda en el proceso. Esto se traduce en mayores tasas de rentabilidad, menores tiempos de recuperación de la inversión y mayores flujos de ahorro, los cuales se acumularan a lo largo de la vida útil del proyecto. Con lo anterior, se impone mayor certidumbre económica y financiera para la evaluación y toma de decisiones en la implementación del esquema de cogeneración.

El aprovechamiento del potencial de cogeneración, ofrece la construcción y el financiamiento de infraestructura eléctrica por parte del sector privado, lo que beneficia a la economía del sector eléctrico, ya que las necesidades de financiamiento son más bajas en los proyectos de cogeneración, debido a la diferencia de costos medios (Cristerna, 1998) y (CRE, 1999) entre el MWe producido en forma convencional y por cogeneración.

Por parte de la tecnología de cogeneración, la más reciente es la micro cogeneración, que es resultado del desarrollo de turbinas de gas a baja capacidad y con un gran mercado en las zonas residenciales; así como la gasificación integrada a ciclo combinado (IGCC), lo que aprovecha combustibles de desecho.

Por sus amplias ventajas, la cogeneración ha logrado la atención de las empresas y los gobiernos, lo cual representa un pronto desarrollo en muchos países. Esta importancia que le han concedido, queda demostrada con la formación de asociaciones y grupos de trabajo para su desarrollo y promoción, por ejemplo, el Grupo Europeo de Cogeneración (GEC), que es el encargado de promover la cogeneración en la Unión Europea (UE). Este grupo trabaja con cuatro temas: ambiental, económico, político y comercial.

A pesar del crecimiento de la cogeneración en muchos países, en México no se ha iniciado este proceso. Desde el primer intento de desarrollo a finales de los años setenta y pasando por la reforma de 1992, se estima (León, 2001) que se han construido y están en operación 190 MWe por cogeneración en la industria privada (1997). La autogeneración en la industria nacional (BNE, 1999), incluyendo Pemex a 1998, es de 3246 MWe, pero no existe información que permita excluirla en cogeneración y convencional; sin embargo, desde que se modificó la ley en 1992 y se abrió el mercado eléctrico a la participación de los particulares en las modalidades de productor independiente, autoabastecimiento, cogeneración, importación y exportación, su crecimiento (León, 2001) se ha manifestado a un ritmo promedio anual muy bajo de $0.17 \%$ con una participación en la capacidad nacional casi insignificante de $0.54 \%$, debido fundamentalmente a que el marco legal dentro del cual se planeó el desarrollo del autoabastecimiento, ya sea por cogeneración o convencional, sufrió grandes deficiencias legales y lagunas jurídicas al no considerar los tiempos y necesidades de estabilidad económica y política, así como de certidumbre legal y falta de sensibilidad para percibir los aspectos importantes que fomentan el desarrollo de esta actividad.

Por tanto, las diversas opiniones convergen en considerar que el crecimiento de la cogeneración podría ser mucho mayor, tomando en cuenta el potencial de cogeneración a 1995 de entre 7586 y 14229 MW, de acuerdo a la más reciente estimación de la Comisión Nacional para el Ahorro de la Energía (CONAE, 1996).

\section{Desarrollo}

En este trabajo la discusión se desarrolla en tres partes, en la primera se plantea el estado que guarda esta 
técnica en el país y un análisis de los requerimientos de desarrollo, asimismo, en la segunda parte se estima el potencial teórico de cogeneración industrial, con base en la información de consumo de combustibles en las diferentes ramas industriales, proporcionada por los balances energéticos nacionales, así como estimaciones sobre eficiencias promedio de los equipos comerciales que se utilizan y los procesos empleados para generar los requerimientos térmicos. Finalmente, en la tercera parte se establecen las ventajas económicas y ambientales, a través de escenarios de aprovechamiento. Éstos toman en cuenta supuestos plausibles de crecimiento de la economía, la evaluación de las necesidades energéticas de la industria y los cambios institucionales.

Para cada escenario se estima el beneficio económico y se calcula el impacto favorable que se tendría sobre el programa de obras del sector eléctrico nacional, evaluando la reducción que resultaría en el consumo de combustibles y, por lo tanto, en las emisiones contaminantes.

\section{Estado del aprovechamiento}

A partir de las reformas a la ley de Servicio de Energía Eléctrica y su reglamento de 1972 y 1992 , se anexaron a la modalidad de autoabastecimiento las opciones de cogeneración, pequeña producción y producción independiente. Hasta 1995, la Secretaría de Energía pensaba que una parte significativa de los requerimientos de capacidad del sistema de generación entre 1997-2006 podían ser satisfechos mediante proyectos de producción independiente, autoabastecimiento y excedentes de cogeneración.

El desarrollo para el aprovechamiento del potencial de cogeneración en México es muy bajo, esta situación es resultado de una falta de voluntad política, así como de la ineficiencia legal para planear el marco jurídico de desarrollo en dicha actividad, esto se ha traducido en una falta de motivación por parte de la industria establecida para instalar o modificar sus sistemas de generación de energía eléctrica o térmica.

Dentro de la autogeneración (CRE, 1999), la cogeneración representa para 1993, aproximadamente un $19 \%$ y para 1999 , un $21 \%$. Con la introducción de la nueva incertidumbre generada por la propuesta de reestructuración del sector eléctrico (1999 y 2001) se espera que la tendencia de crecimiento moderado se estanque, ya que no se considera la potencialidad de la cogeneración en el desarrollo de la infraestructura eléctrica del país en forma importante, relegándola a una medida de ahorro de energía, por lo que nunca se logrará llevar la cogeneración a niveles internacionales medios de participación de 10 - 12 \% y mucho menos de los de Europa del Norte de $35 \%$ en la generación total del país.

\section{Requerimientos de mercado eléctrico}

Las cifras oficiales de la demanda de energía, se manejan en función del crecimiento de la economía y la población, así como de las plantas en restauración y el margen de reserva, en donde la CFE ha fijado un $27 \%$ como ó ptimo para el tamaño y tipo de sistema en el país.

Conociendo los datos de crecimiento bajo estos parámetros, así como la visión del gobierno, se establece un escenario de incremento en las ventas de energía de entre $5.43 \%$ y 7.3\%. La generación histórica de los últimos 8 años muestra un aumento en la generación decreciente y en el orden de 5 a $5.6 \%$, período que abarca la reforma de 1992 . Por ello, es más probable que el escenario oficial válido sea de $5.48 \%$ y que esté a sociado a un crecimiento de $4.3 \%$ de la economía.

Utilizando un indicador ${ }^{1}$ que señale la relación entre el crecimiento de la capacidad de generación $y$ el requerimiento de generación (IPE = $\mathrm{MW} / \mathrm{TWh}$ ), surge un índice con una pendiente decreciente, ya que disminuye su valor por el descenso en el incremento del numerador respecto al denominador en diferente proporción. Es decir, la capacidad no crece en la misma medida que las ventas (Figura 1).

La relación histórica en cuanto al crecimiento de la capacidad y las ventas de energía, así como el proyectado, muestran su nivel de crecimiento dependiendo de la otra. Si la relación se mantiene constante en un valor, esto quiere decir que ambas

1 Índice Potencia Energía (IPE) 


\section{TENDENCIA CAPACIDAD/VENTAS \\ y Equilibrio his tórico y esperado}

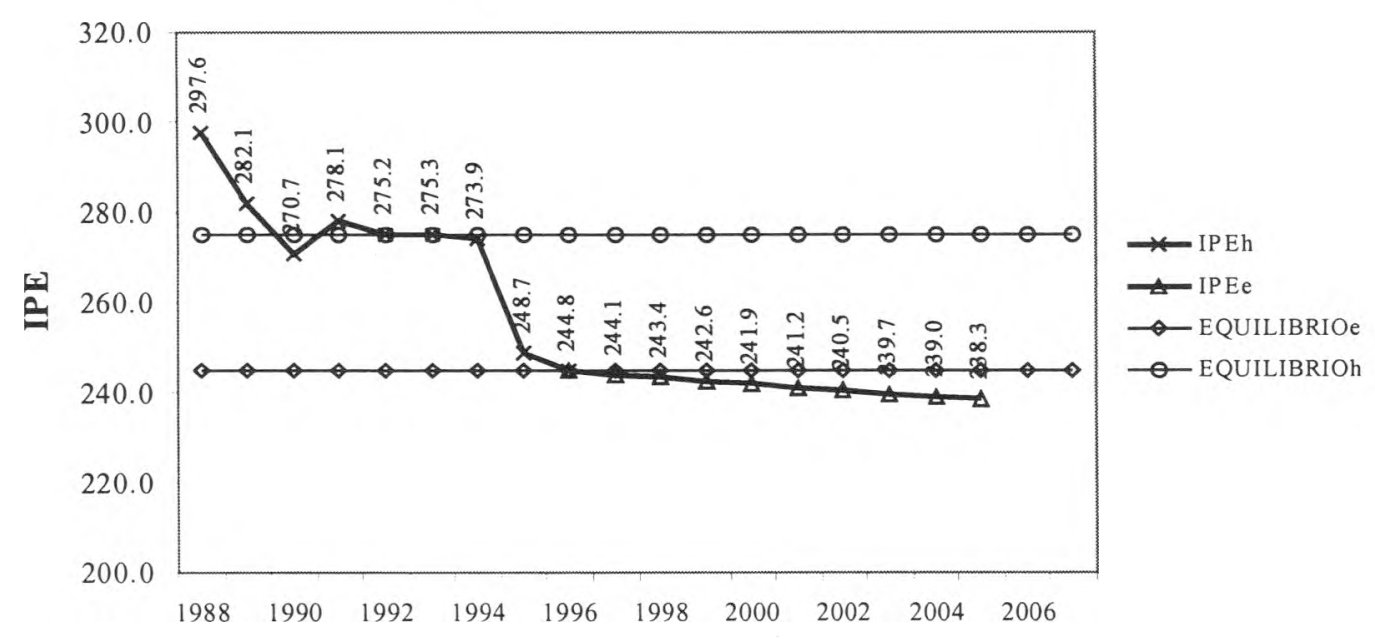

Fuente: Elaboración propia con datos de los Balances Nacionales de Energía (1997) y Prospectiva del Sector Eléctrico Nacional (1997).

Figura 1

crecen al mismo nivel, pero si el valor crece o decrece indica que en la relación una de las dos no mantiene el mismo comportamiento. Tal situación proyectada al 2003, estaría acercando al sistema eléctrico nacional a un déficit de suministro y capacidad para atender el crecimiento del país ${ }^{2}$.

En la figura 1, se observa que el gobierno de Salinas recibió al sector eléctrico con un valor de 368 , y lo entregó con 275 , es decir, disminuyó la capacidad de reserva del sistema eléctrico. En el gobierno de Zedillo para el año de 1998, decreció aún más, con un valor de 244 que representó en ese año el $27 \%$ de capacidad de respaldo instalada.
En forma gráfica se observa una tendencia decreciente de la capacidad instalada respecto a las ventas. En 1994, se muestra un desplome en la inversión para la construcción de infraestructura eléctrica, respecto a las ventas, y al mismo tiempo, al crecimiento de la demanda. Tal situación se ve frenada con la proyección del gobierno al 2007, pero continua en decrecimiento.

Esta política de deterioro de la capacidad respecto a las ventas, tendría repercusiones económicas y financieras de $37,446 \mathrm{mdd}$ por los próximos diez años, es decir, 3746 mdd/año, según el gobierno federal.

Tabla 1. Requerimiento oficial de inversión en el Sistema Eléctrico Nacional 1998-2007

\begin{tabular}{|c|c|c|c|c|c|}
\hline Crecimiento & Costo & $\mathrm{mdd} / \mathrm{MWe}$ & MWe a 1998 & MWe a 2007 & MWe adicionales \\
\hline $5.483 \%$ & Promedio & & 35255 & 56998 & 21743.4 \\
\hline Generación & $\$ 0.751$ & & & $\$ 16,329$ & \\
\hline Transmisión & $\$ 0.422$ & & & $\$ 9,176$ & \\
\hline Distribución & $\$ 0.250$ & & & $\$ \quad 5,427$ & \\
\hline Mantenimiento & $\$ 0.293$ & & & $\$ 6,364$ & \\
\hline Ingeniería & $\$ 0.007$ & & & $\$ 150$ & \\
\hline Consumido & $\$ 1.722$ & & & $\$ 37,446$ & $\mathrm{Mdd} / \mathrm{MWe}$ \\
\hline
\end{tabular}


El cuestionamiento actual, es de dónde salen los 3746 mdd por año o los 2174.3 MWe por año de capacidad adicional; en principio, los dólares pueden salir de tarifas apegadas a precios de mercado, de reducción de subsidios, del cambio de prioridades o de productores independientes (PIE). Y los MWe del autoabastecimiento y del potencial nacional de cogeneración por desarrollar. Parte de los $21743 \mathrm{MWe}$ podrían salir de la potencialidad que la cogeneración ofrece, esta opción no es nueva, ya que en otros países se ha realizado, además de que significa mayor eficiencia económica y asignativa, así como el menor impacto ambiental que la forma convencional.

\section{Estimación del potencial de cogeneración}

En el Balance Nacional de Energía de 1997, la autoproducción de energía del sector privado, eliminando a Pemex, fue de $1411 \mathrm{MWe}$, de los cuales no existió desagregación que diera a conocer la proporción de cogeneración y autoproducción.

La CRE reporta a diciembre de 1997, 1343.3 MW y a diciembre de 1999, 1824.1 MW de autoproducción en proyectos autorizados y en vías de implantación. La CRE menciona que el $71 \%$ de los permisos otorgados en 1997 de forma general, están en operación, por lo que se podría a proximar más el dato de la capacidad privada de generación al quedar en (1343.3)(0.71) =953.74 MWe. Por otro lado, los permisos otorgados para la generación privada, con una proporción del $21 \%$ son proyectos para cogeneración, por lo que se podría estimar una potencia instalada de plantas de cogeneración de (953.74)(0.21) = 190.74 MWe a 1997 en la industria privada.

El potencial de cogeneración industrial histórico (1992-1997) se obtiene mediante una clasificación de procesos industriales típicos de sistema superior e inferior, éste se complementa con estadísticas de consumos de combustibles industriales para generación de vapor y temperatura (León, 2002), también con base en las eficiencias promedio de generación de vapor y temperatura que ofrecen los equipos comerciales y los rangos teóricos de recuperación de energía que otorgan los equipos de cogeneración. Éstos se basan en el criterio del abasto del $100 \%$ de la demanda térmica y del factor de planta.

Este potencial de cogeneración basado en el consumo de combustible, es afectado por la intensidad del mismo, que a su vez, depende del nivel de producción o factor de planta; por lo que el potencial de cogeneración que utiliza toda la capacidad instalada o el $100 \%$ de la capacidad de planta, dará como resultado el incremento del potencial. Con estos resultados se determinan las bases de inicio y crecimiento histórico promedio del potencial, que refleja una proyección a diez años.

Tabla 2. Potencial de cogeneración Sector Industrial MWe

\begin{tabular}{|c|c|c|c|c|c|}
\hline SECTOR & SISTEMA & SISTEMA & TOTAL & INCREMENTO & CRECIMIENTO \\
\hline INDUSTRIAL & SUPERIOR & INFERIOR & NACIONAL & & MEDIO \\
\hline 1992 & $7,671.0$ & 960.9 & $8,631.9$ & & \\
\hline 1993 & $8,432.7$ & 983.7 & $9,416.3$ & $8.3 \%$ & \\
\hline 1994 & $8,672.0$ & $1,036.1$ & $9,708.1$ & $3.0 \%$ & $7.97 \%$ \\
\hline 1995 & $9,630.0$ & $1,063.2$ & $10,693.1$ & $9.2 \%$ & \\
\hline 1996 & $10,916.6$ & $1,141.0$ & $12,057.6$ & $11.3 \%$ & \\
\hline
\end{tabular}

Fuente: Elaboración propia

2 Una tasa de crecimiento de la capacidad de generación de 7.44\% promedio (asociado a un PIB de 5.6\%) revertiría moderadamente la tendencia decreciente y ubicaría al IPEe en 249 en vez de 238 al año 2007, con el crecimiento esperado oficial de 5.43\%. 


\section{Estimación del crecimiento del potencial teórico}

El potencial histórico de cogeneración industrial se toma como base para realizar una proyección de su crecimiento, por tanto, el objetivo en materia de desarrollo de fuentes alternas para la construcción de infraestructura eléctrica, podría enfocarse en la utilización de este potencial como forma de reducción de costos y contaminación, así como para preservar los recursos energéticos. En la figura 2 se proyecta el crecimiento del potencial de cogeneración en función del crecimiento histórico, en donde se obtiene un potencial teórico medio de 28,000 MWe al año 2007.

\section{Aprovechamiento del potencial industrial de cogeneración viable}

De algunos estudios de viabilidad realizados en la industria nacional durante los años de 1994 al 2000 , se reportan los siguientes niveles de tasas internas de rendimiento, reconociendo que los proyectos más viables son los que presentan el mayor rendimiento económico, criterio fundamental desde el punto de vista industrial.

Como el criterio de desición es el beneficio económico, el potencial con rendimientos clasificados como "poco rentables" no podrá ser aprovechado ni atractivo para la industria, a menos que el nivel de riesgo país en

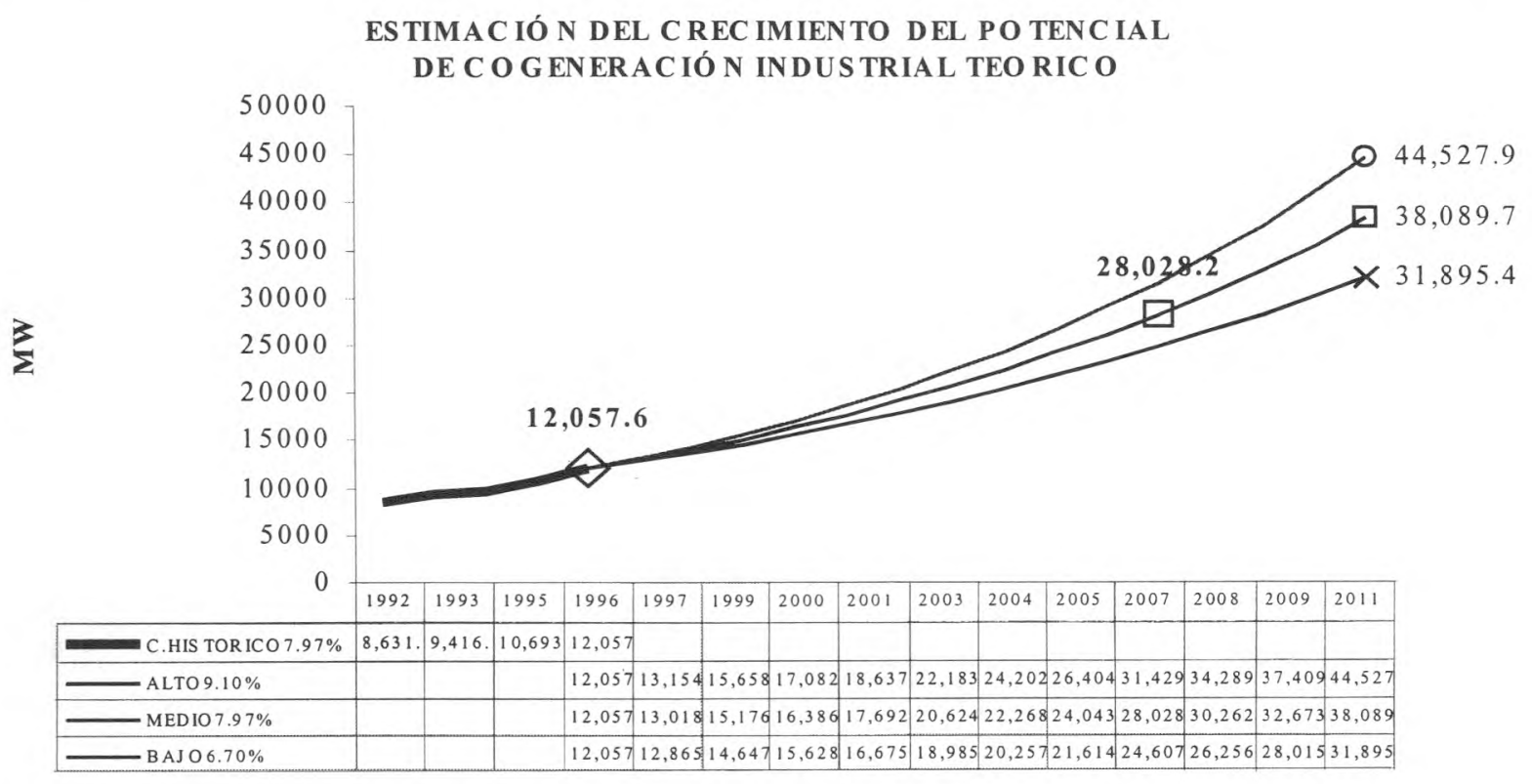

Fuente: Elaboración propia.

Figura 2

Tabla 3. Viabilidad económica en los proyectos de cogeneración

TIR

Excelente
Muy buena
Buena
Regular
Poco rentable

Poco rentable

RANGO DE CALIFICACIÓN

$43.0 \%$

$25.0 \%$

$15.0 \%$

$10.0 \%$

$0 \%$
$133.0 \%$

$42.9 \%$

$24.9 \%$

$14.9 \%$

$9.9 \%$
\% CASOS PRÁCTIICOS OUE CAEN EN ESTA CLASIFICACIÖN

\section{$3.85 \%$}

$15.38 \%$

$38.46 \%$

$34.62 \%$

$7.69 \%$

Fuente: Elaboración propia con 250 datos de CONAE (1994), 6 de consultorías (1996-1998) y 2 propios (1998-2000).

3 La práctica en la evaluación de proyectos de cogeneración en el país con tasas de riesgo de 10 - 12 \% ha mostrado que los esquemas de financiamiento por proveedores toman como parámetro de decisión tasas de rendimiento por encima del 18\%. Aunque en la opinión de especialistas financieros como Luis Fernández para las condiciones del país, ésta sería aceptable si se ubicara cuando menos en $15 \%$ real. 
materia de tasas de financiamiento se reduzca, y con ello, una parte de este potencial no viable con tasas de riesgo de $10-15 \%$ pase a ser variable.

Proyectando el crecimiento del potencial de cogeneración en función de los niveles de viabilidad mostrados en la tabla 3 , se logrará un potencial teórico medio de 25,872 MW al 2007. Por lo tanto, el crecimiento de los proyectos de cogeneración deberá tratar de sumar estevalor y desaprovechar la parte del potencial cuya rentabilidad está por debajo del $10 \%$ (Figura 3).
Ventajas económicas y ambientales

La evaluación de las ventajas económicas se basa en la información anterior y en función de las metas de desarrollo, donde se estima el crecimiento promedio anual de la cogeneración. También es muy importante reiterar que México es un país pobre, por tanto, para planear su desarrollo deberá evitarse el desperdicio de recursos económicos y naturales. Por tal motivo, es necesario considerar cuidadosamente las necesidades de desarrollo, ya que la cogeneración desplaza la capacidad instalada, que pudiera traducirse en capacidad osciosa como la experiencia sufrida en los EU.

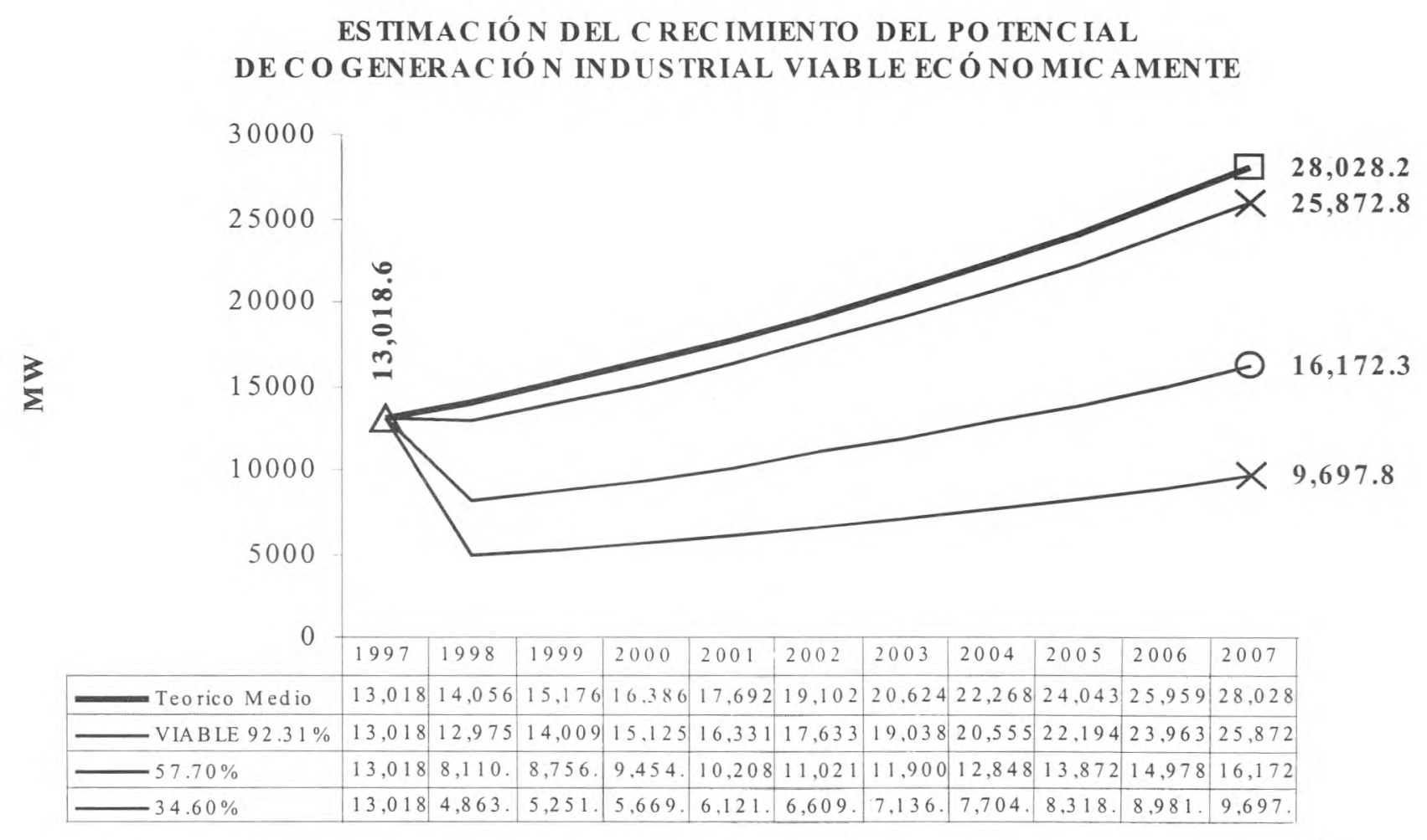

Fuente: Elaboración propia.

Figura 3

Tabla 4. Indicadores definidos

\begin{tabular}{lcccc}
\multicolumn{1}{c}{ VALORES OFICIALES } & ALTO & MEDIO & BAJO & CONVENCIONAL \\
Consumo nacional de energía \% & 5.48 & 5.48 & 5.48 & 5.48 \\
Capacidad adicional MW & 21743 & 21743 & 21743 & 21743 \\
Capacidad de generación MW & 35225 & 35225 & 35225 & 35225 \\
PIB \% & 4.3 & 4.3 & 4.3 & 4.3 \\
\hline \hline
\end{tabular}


Tabla 4. Indicadores definidos (continuación)

\begin{tabular}{lcccc}
\hline \multicolumn{1}{c}{ VALORES OFICIALES } & ALTO & MEDIO & BAJO & CONVENCIONAL \\
Actividad Industrial \% & 5.3 & 5.3 & 5.3 & 5.3 \\
Crecimiento Poblacional \% & 1.3 & 1.3 & 1.3 & 1.3 \\
Crecimiento de la vivienda \% & 2.5 & 2.5 & 2.5 & 2.5 \\
Crecimiento de nivel tarifario \% & 3 & 3 & 3 & 3 \\
Crecimiento de las ventas & 6.1 & 6.1 & 6.1 & 6.1 \\
Costo medio del MW consumido para la CFE & 1.722 & 1.722 & 1.722 & 1.722 \\
Costo medio del MW consumido para cogeneración mdd & 1.166 & 1.166 & 1.166 & 1.166 \\
Crecimiento de la demanda auto abastecida convencional & 7.7 & 7.7 & 7.7 & 7.7 \\
Combustible gas & 68.5 & 68.5 & 68.5 & 68.5 \\
Tecnología de generación CFE & CC & CC & CC & CC \\
Tecnología de cogeneración & CI y TG & CI y TG & CI y TG & CI y TG \\
\hline \hline
\end{tabular}

En esta proyección se hacen las siguientes consideraciones generales:

1. Se basa en un modelo tipo $(1+i)^{n}$.

2. La curva de crecimiento de la CFE más la de la cogeneración deberá ser igual a la del país.
3. La capacidad por cogeneración proyectada no deberá ser más grande que la del potencial viable disponible, considerando excedentes de cogeneración.

4. El nivel de crecimiento se proyecta en dos etapas de cinco años cada una.

Tabla 5. Acciones a implementar

\begin{tabular}{|c|c|c|c|c|}
\hline ACCIONES ${ }^{4}$ & $\checkmark$ & Modifica & $x$ & Sigue igual \\
\hline Crecimiento del potencial de cogeneración \% & 7.97 & 7.97 & 7.97 & 7.97 \\
\hline Eliminar incertidumbre en la organización del sector eléctrico & $\checkmark$ & $\checkmark$ & $\checkmark$ & $x$ \\
\hline Eliminar del marco legal la sujeción a despacho & $\checkmark$ & $\checkmark$ & $\checkmark$ & $x$ \\
\hline Eliminar del marco legal la limitación de venta de exedentes & $\checkmark$ & $\checkmark$ & $x$ & $x$ \\
\hline $\begin{array}{l}\text { Definir mecanismo para la determinación de los precios de venta } \\
\text { de exedentes }\end{array}$ & $\checkmark$ & $\checkmark$ & $\checkmark$ & $x$ \\
\hline Incluir el reconocimiento del costo por capacidad & $\checkmark$ & $\checkmark$ & $\checkmark$ & $x$ \\
\hline $\begin{array}{l}\text { Que el costo del porteo para cogeneración sea el costo de CFE y } \\
\text { para el autoabastecimiento convencional al costo de CFE más una } \\
\text { utilidad razonable }\end{array}$ & $\checkmark$ & $x$ & $x$ & $x$ \\
\hline Desarrollo suficiente de la infraestructura de gas & $\checkmark$ & $\checkmark$ & $x$ & $x$ \\
\hline $\begin{array}{l}\text { Aceptación de venta de exedentes naturales a costo evitado y el } \\
\text { resto a precios de mercado }\end{array}$ & $\checkmark$ & $x$ & $x$ & $x$ \\
\hline $\begin{array}{l}\text { Cambio de mentalidad en la cultura empresarial hacia la } \\
\text { innovación }\end{array}$ & $\checkmark$ & $\checkmark$ & $\checkmark$ & $x$ \\
\hline Tasa por riesgo \% & 5 & 9 & 10 & 15 \\
\hline Nivel de viabilidad en proyectos de cogeneración \% & 92.3 & 66 & 57.7 & 34.6 \\
\hline
\end{tabular}

Fuente: Elaboración propia.

4 Acciones identificadas y propuestas por el autor tomando en cuenta los siguientes puntos: a) Aquí se introduce una distinción en la demanda, ya que el autoabastecimiento por cogeneración es diferente al de sistemas convencionales, así como sus intereses y naturaleza. b) Datos obtenidos en la Gerencia de Programación de Sistemas Eléctricos de la CFE (1999). c) No se utiliza el valor obtenido con los datos de la CRE de $0.517 \mathrm{mdd}$, el cual, sin duda hará mayores las ventajas económicas; sin embargo, se emplea un valor medio comercial obtenido con fabricantes y desarrolladores en el país, al 2000. d) Algunos de estos puntos son tomados de la XIV y XVIII Reunión de subcomisión para promover proyectos de cogeneración, CONAE. Con base a exposiciones del Dr. Antonio Souza, Director de inversión. Protego 2000. 
DOI: http://dx.doi.org/10.22201/fi.25940732e.2003.04n1.004

Tabla 6. Proyección con diferentes niveles de crecimiento de la cogeneración industrial

\begin{tabular}{ccccccccc}
\hline \hline ETAPAS & $1998-2003$ & $2004-2007$ & $1998-2003$ & $2004-2007$ & $1998-2003$ & $2004-2007$ & $1998-2003$ & $2004-2007$ \\
$\begin{array}{c}\text { Tasa de } \\
\text { crecimiento }\end{array}$ & $79.80 \%$ & $21.20 \%$ & $65.15 \%$ & $22.59 \%$ & $32.00 \%$ & $28.00 \%$ & $1.60 \%$ & $1.90 \%$ \\
Escenario & ALTO & \multicolumn{2}{c}{ MEDIO } & & BAJO & CONVENCIONAL \\
\hline
\end{tabular}

Fuente. Elaboración propia.

Tabla 7. Costos para los diferentes niveles de crecimiento mdd

\begin{tabular}{lcccc}
\hline \multicolumn{1}{c}{ ACTORES } & ALTO & MEDIO & BAJO & CONVENCIONAL \\
\hline Costo CFE & $\$ 14,899$ & $\$ 22,942$ & $\$ 33,220$ & $\$ 37,395$ \\
Costo cogeneración & $\$ 15,814$ & $\$ 9,824$ & $\$ 2,865$ & $\$ 38$ \\
Costo total al país & $\$ 30,713$ & $\$ 32,766$ & $\$ 36,085$ & $\$ 37,433$ \\
\hline \hline
\end{tabular}

Fuente. Elaboración propia.

Tabla 8. Ahorro en la inversión

\begin{tabular}{lcccc}
\hline \hline \multicolumn{1}{c}{ PROYECCIÓN } & ALTO & MEDIO & BAJO & CONVENCIONAL \\
\hline A CFE & $60.16 \%$ & $38.65 \%$ & $11.16 \%$ & $0.00 \%$ \\
A la cogeneración & $0.00 \%$ & $37.88 \%$ & $81.88 \%$ & $99.76 \%$ \\
Al país & $17.95 \%$ & $12.47 \%$ & $3.60 \%$ & $0.00 \%$ \\
\hline
\end{tabular}

Fuente. Elaboración propia.

\section{Efecto económico}

La proyección permite estimaciones para cuatro escenarios de niveles de crecimiento, de montos de inversión y ahorros. Estos resultados se resumen en las tablas 6,7 y 8 .

Se resalta la diferencia entre los resultados del escenario convencional (actual), y cualquiera de los otros.

La tasa de crecimiento de la cogeneración en la próxima década, presenta una relación de interdependencia con la tasa de crecimiento de la CFE, ya que ambas (y con la participación del autoabastecimiento y las importaciones) deberán cubrir la demanda del país sin excederse; por esta razón, el nivel de crecimiento de la cogeneración debe implicar una disminución o estabilidad en el crecimiento de la CFE.

La tabla 7 muestra posibles relaciones de costos de construcción en la infraestructura eléctrica del país durante la próxima década, así como sus diferentes montos en función de algunas tasas de crecimiento de la cogeneración. De esta forma, se observa que el costo mayor se tiene cuando la cogeneración crece a los niveles actuales, y es mínima cuando crece a niveles de $79 \%$ y $21 \%$, sin sobrepasar su potencial.

Proyectando estas posibles ventajas con niveles de crecimiento de la cogeneración, se desprenden algunos resultados que muestran los niveles de beneficio para los actores y para el país.

El beneficio total es función del nivel de participación de la cogeneración al satisfacer la 
demanda nacional que se pueda lograr. De estos resultados podemos desprender que la cogeneración puede ofrecer importantes posibilidades de participación y apoyo para la CFE, principalmente en la construcción y abasto de la energía eléctrica que consumirá el país en los próximos diez años. Esta situación es ventajosa para los actores que deben protagonizar este impulso, así como el planteamiento y mantenimiento de la cogeneración.

\section{Efecto ambiental}

El consumo de combustible en la industria está ligado al crecimiento industrial del producto bruto, por lo que mantendrá una relación de crecimiento similar, a excepción de que existan cambios en la intensidad energética, debidos a variaciones estructurales, condiciones de operación o factor de planta.

Tomando en cuenta estos datos y los índices de emisiones, es posible estimar los escenarios de crecimiento en la emisión y su afectación con la inserción del crecimiento de la cogeneración en la industria nacional (Figura 4).

La reducción de emisiones contaminantes es el resultado del bajo consumo de combustible, el cual representa para los principales contaminantes las cantidades de la tabla 9.

Esta imagen gráfica, ubicaría un potencial de reducción del $21.49 \%$ en las emisiones que hace la industria, respecto a la proyección convencional sin crecimiento de la cogeneración. Este desarrollo es equivalente a una disminución a nivel país de $7.7 \%$.

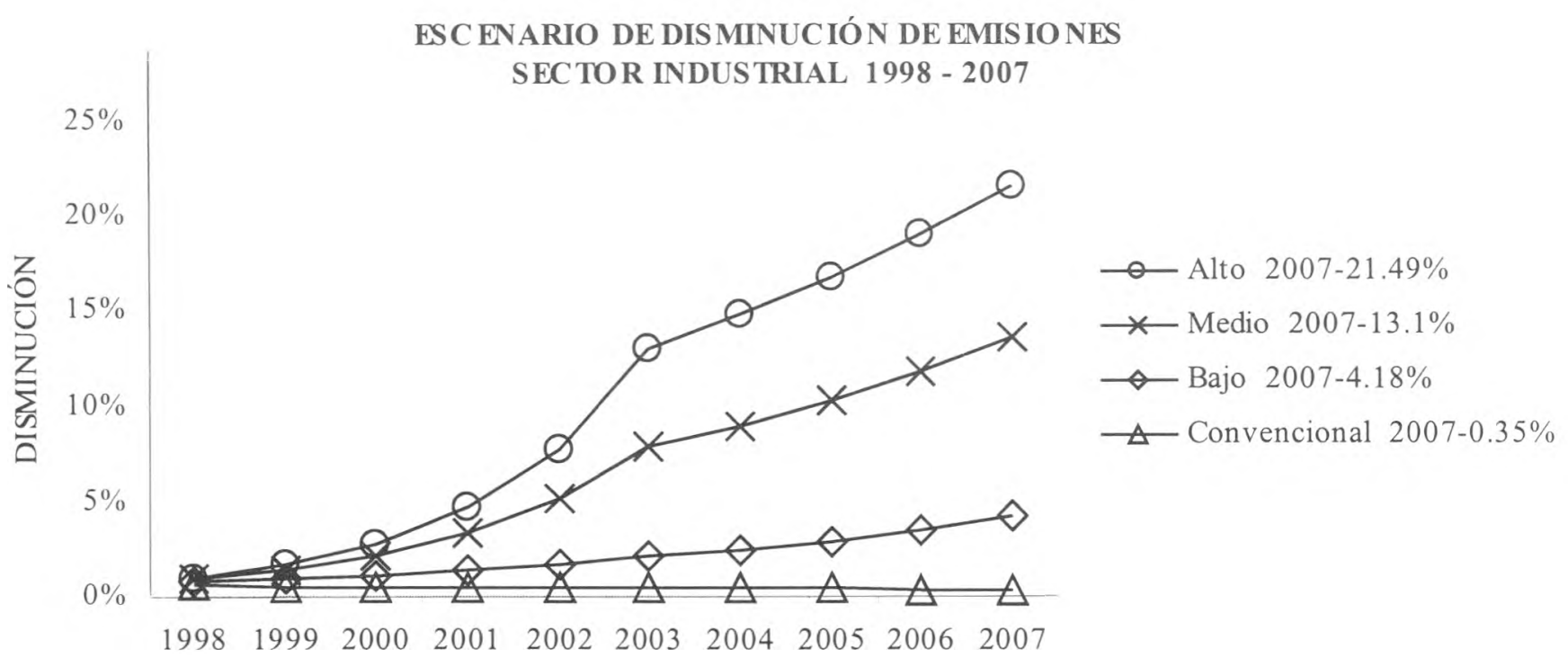

Fuente: Elaboración propia

Figura 4

Tabla 9. Equivalente en Ton/año de reducción de emisiones

\begin{tabular}{ccccccccccc}
\hline $\begin{array}{c}\text { ALTO Vs } \\
\text { CONVENCIONAL }\end{array}$ & 1998 & 1999 & 2000 & 2001 & 2002 & 2003 & 2004 & 2005 & 2006 & 2007 \\
\hline $\begin{array}{c}\text { Aumento en la } \\
\text { reducción }\end{array}$ & $76.8 \%$ & $213.2 \%$ & $454.5 \%$ & $881.8 \%$ & $1639.8 \%$ & $2968.6 \%$ & $3550.0 \%$ & $4242.2 \%$ & $5068.0 \%$ & $6053.1 \%$ \\
$\mathrm{CO}_{2}$ & 1220.30 & 2195.12 & 3945.52 & 7090.54 & 12750.88 & 22925.97 & 27789.38 & 33677.42 & 40817.77 & 49473.71 \\
$\mathrm{SO}_{2}$ & 9.1 & 16.3 & 29.3 & 52.7 & 94.7 & 170.3 & 206.4 & 250.1 & 303.2 & 367.4 \\
$\mathrm{NO}_{x}$ & 0.7 & 1.2 & 2.1 & 3.8 & 6.8 & 12.3 & 14.9 & 18.1 & 21.9 & 26.5 \\
\hline
\end{tabular}




\section{Conclusiones}

Se estiman costos de 30,713 mdd contra 37,433 mdd entre el escenario alto y el convencional, lo que representaría un potencial de ahorro de casi 7000 mdd para el país en divisas, las cuales no se gastarían ni saldrían del país. Asimismo, la industria financiaría 15,814 mdd y la empresa eléctrica 14,899 mdd para tener el mismo nivel de infraestructura eléctrica, en contraposición del convencional, en donde la empresa eléctrica gastaría 37,395 mdd y la industria $38 \mathrm{mdd}$. La reducción de emisiones contaminantes que se podría obtener es de $2007-21.49 \%$ para el escenario alto y de 2007-0.35\% \% para el convencional. México no se caracteriza por ser un gran generador de emisiones contaminantes en el ámbito internacional, pero tampoco se caracteriza por ser un país que invierta mucho dinero en programas de mitigación.

Por tanto, se requiere buscar políticamente la inclusión de una forma más protagónica de la cogeneración en este proceso de construcción de la infraestructura eléctrica del país, a través de programas de desarrollo, apoyos, certidumbre económica y legal, estímulos, difusión y la mejora en las condiciones de percepción que los industriales tienen respecto a esta técnica y procedimientos de implementación.

Una muestra de este tipo de medidas ${ }^{5}$, fue realizada por el nuevo gobierno el 11 de mayo del 2001, la cual reformó el reglamento del servicio público de energía eléctrica, permitiendo el $100 \%$ de la capacidad como venta de excedentes eléctricos para las plantas cogeneradoras y el $50 \%$ de la capacidad instalada para la modalidad de autoabastecimiento, ello después de 5 años de su solicitud.

\section{Referencias}

Balances Nacionales de Energía (1997, 1998, 1999).

Cristerna-Ocampo R. (1994). Lineamientos del POSE-RCO/arp.DEPFI, UNAM, México. Reporte del Grupo Europeo de Cogeneración (GEC), (1998). Balances Nacionales de Energía (1997, 1998, 1999).

CFE (1997). Precios internos y externos de referencia de los principales energéticos, período 1970-1996. 8a edición de la Subdirección de Programación, Gerencia de Estudios Económicos. CFE. México.

Comisión Reguladora de Energía (CRE).

$(1998,1999,2000)$. InfoCre, varios reportes. CRE. México.

CONAE (1992, 1995, 1996 y 1998). Memorias de congresos internacionales de cogeneración. México.

CONAE (1996). Potencial nacional de cogeneración 1995. CONAE, México.

Fernández-Montiel M., González-Santaló J.M. (1999). Tendencias tecnológicas en los procesos de combustión en la generación de electricidad. IIE. México.

León de los Santos G. (2002). Ventajas económicas $y$ ambientales del aprovechamiento del potencial industrial de cogeneración en México y condiciones para su viabilidad. Tesis de doctorado, DEPFI, UNAM, México.

Prospectiva del Sector Eléctrico Nacional 1998-2007. (1997). Secretaría de Energía.

Prospectiva del Sector Eléctrico Nacional (PSEN) 1997-2006. (1998). Secretaría de Energía.

Reporte del Grupo Europeo de Cogeneración (GEC), (1998).

\section{Bibliografía sugerida}

CONAE (1993). Primeras jornadas de cogeneración. En: Congresos internacionales de cogeneración en México 1992 . México.

CONAE (2002). Elementos para la exposición de motivos y elaboración de un régimen especial para la cogeneración en el mercado eléctrico mexicano. Mayo 2002.

IDAE (1997). La cogeneración en México y expectativas internacionales. CONAE, México.

OCDE (1998). Projected Costs of Genereting Electricity. Update 1998.

Nieva R., Hernández J.A., Portes E. Alvarez J.M. (1996). Evaluación del potencial nacional de

5 Esta medida fue anulada por el Tribunal Superior de Justicia, mediante la resolución de la controversia constitucional promovida por el senador Manuel Bartlet, presidente de la Comisión de Puntos Constitucionales de la Cámara de Senadores en mayo del 2002. 
cogeneración como opción en la expansión del sistema eléctrico nacional. IIE, México.

Resendiz-Nuñez D. (1994). El sector eléctrico de México. Ed. FCE. México.

\section{Semblanza del autor}

Gabriel León de los Santos. Es ingeniero mecánico egresado con mención honorífica en el año 1993 por la FES-Cuautitlán, UNAM. Realizó la maestría en ingeniería energética también con mención honorífica en 1998 y el doctorado en el área de investigación en economía de la energía en el 2001, en la División de Estudios de Posgrado de la Facultad de Ingeniería de la UNAM. Desde 1999 imparte el curso de termodinámica en la Facultad de Ingeniería. Ha prestado sus servicios en varias empresas del sector privado en las áreas de ventas, servicio, proyectos y mantenimiento, laborando como ejecutivo de ventas para equipo de manejo de materiales y pavimentación, en Mextrac, SA de CV (1993); jefe de servicio de calderas en Selmec Equipos Industriales SA de CV (1994 -1998); gerente de proyectos en Thermo Energía Sistemas y Equipos SA de CV (1999 -2001); y gerente técnico en Mantenimiento General y Comercial SA de CV (2002). 